\title{
ESTUDO HISTOLÓGICO E COMPUTADORIZADO DAS ÁREAS COM CÉLULAS PARIETAIS E PRINCIPAIS NO ESTÔMAGO DE RATOS WISTAR TRATADOS COM PANTOPRAZOL E "N-NITROSO-N-METHYLUREA"(NMU) ${ }^{1}$
}

\author{
Iure Kalinine Ferraz de Souza ${ }^{2}$ \\ Alcino Lázaro da Silva ${ }^{3}$ \\ Atsunobu Misumi ${ }^{4}$ \\ Daniela Savi $^{5}$ \\ Denílson Santos Custódio ${ }^{6}$ \\ Frederico Fernandes Ribeiro Maia $^{6}$ \\ Giovanne Guilherme Coutinho Canela ${ }^{6}$ \\ Carlos Henrique Cardoso de Oliveira ${ }^{6}$
}

Souza IKF, Lázaro da Silva A, Misumi A, Savi D, Custódio DS, Maia FFR, Canela GGC, Oliveira CHC. Estudo histológico e computadorizado das áreas com células parietais e principais no estômago de ratos Wistar tratados com pantoprazol e "N-Nitroso-N-Methylurea" (NMU). Acta Cir Bras [serial online] 2002 Jul-Ago;17(4). Disponível em URL: http://www.scielo.br/acb.

RESUMO - O uso prolongado dos inibidores da bomba de prótons tem sido considerado uma condição de risco para o desenvolvimento de gastrite atrófica e tumores gástricos. Objetivo: Estudar o efeito do uso de pantoprazol (PTZ) e carcinogênese pela "N-Nitroso-N-Methylurea" (NMU), por 15 semanas, sobre o estômago glandular de ratos Wistar, pela análise histológica e computadorizada das áreas com células parietais (AP), principais (AZ) e da mucosa não oxíntica (ANO), além do estudo das alterações histopatológicas identificadas. Métodos: Quarenta ratos Wistar machos foram distribuídos em 4 grupos: G1 (controle), G2 (NMU+PTZ), G3 (PTZ) e G4 (NMU). O pantoprazol foi administrado $2 \mathrm{x} / \mathrm{semana}(14 \mathrm{mg} / \mathrm{kg}$ de peso, i.p.) e a NMU oferecida, $a d$ libitum, diluída na água de beber $(100 \mu \mathrm{g} / \mathrm{ml})$. Após o estudo histológico AP, AZ e ANO foram determinadas por análise computadorizada das imagens dos estômagos, utilizando o programa "ImageJ 1.19z". Resultados: Mostraram redução da AP e aumento da ANO, em G2, G3 e G4 $(p<0,001)$. Foram encontrados casos de atrofia, inflamação aguda e inflamação crônica, em número que impediu comparação estatística entre os grupos estudados. Conclusão: O uso contínuo de pantoprazol (i.p.), por 15 semanas, reduziu a área com células parietais e aumentou a área de mucosa não oxíntica no estômago glandular de ratos Wistar machos. O mesmo aconteceu no grupo de animais que receberam NMU isoladamente ou em associação com o pantoprazol.

DESCRITORES: Pantoprazol. Metilnitrosouréia. Neoplasias gástricas. Gastrite. Atrofia.

1. Trabalho realizado no Curso de Pós-Graduação em Cirurgia da Faculdade de Medicina da UFMG, e nos Laboratórios de Técnica Cirúrgica e Cirurgia Experimental, Histologia e Patologia da Faculdade de Ciências Médicas de Minas Gerais.

2. Doutor em Técnica Cirúrgica e Cirurgia Experimental pela Faculdade de Medicina da UFMG.

3. Professor Titular de Cirurgia do Aparelho Digestivo da Faculdade de Medicina da UFMG.

4. Professor of the Second Department of Surgery, Kumamoto University, Japan.

5. Médica Patologista do Serviço de Anatomia Patológica do Hospital Semper de Belo Horizonte.

6. Acadêmicos do Curso de Medicina da Faculdade de Ciências Médicas de Minas Gerais. 


\section{INTRODUÇÃO}

Estudos epidemiológicos têm sugerido que fatores como; senilidade, prévio comprometimento da mucosa e fatores ambientais e dietéticos teriam mais influência que aqueles genéticos na patogênese do câncer gástrico. A gastrite crônica atrófica (GCA), com a intestinalização da mucosa gástrica que a acompanha, predispõe ao desenvolvimento do câncer gástrico, pois o epitélio metaplásico tem tendência a maior proliferação celular, enquanto que, por outro lado, pelo seu deficiente poder de inativação protéica, propicia maior contato das substâncias carcinogênicas ingeridas com a mucosa ${ }^{1}$.

Estudos experimentais têm indicado um risco aumentado para o desenvolvimento do câncer gástrico, após o uso prolongado de inibidores da $\mathrm{H}^{+} \mathrm{K}^{+}$/ATPase (IBP). A maior parte destes estudos refere-se à ação do omeprazol, e poucos são os trabalhos que utilizaram outros IBP, como o lanzoprazol e o pantoprazol ${ }^{2,3}$. O mecanismo carcinogênico estaria relacionado com a produção aumentada de peptídeos tróficos, como a gastrina, em resposta a hipocloridria terapêutica prolongada, além de metaplasia intestinal e hiperplasia de células endócrinas. No estômago do rato esta ação trófica determina o aparecimento de tumores carcinóides e aumento da população de células "Entero-Cromafin-Like" (ECL), além de estar relacionada com o aparecimento do adenocarcinoma e carcinoma de células escamosas induzidos por compostos $\mathrm{N}$-nitrosos ${ }^{4}$.

Nitrosaminas formadas pela ação bacteriana em estômagos hipoclorídricos tem sido consideradas como um importante fator no desenvolvimento do câncer gástrico. O uso prolongado dos IBP reduz a acidez gástrica o suficiente para permitir a proliferação bacteriana, sobretudo de Escherichia coli e Pseudomonas $s p$. Em estômagos hipoclorídricos essas bactérias promovem uma eficiente redução de nitratos em nitritos, catalisando o processo de Nitrosação com conseqüente formação endógena de compostos N-nitrosos. Outro importante aspecto a ser considerado é a redução significativa do índice ácido ascórbico / nitritos no suco gástrico de pacientes usuários de omeprazol (40mg/ dia por quatro semanas), o que sugere um risco aumentado para o câncer gástrico, uma vez que esta relação regularia a síntese de compostos $\mathrm{N}$-nitrosos potencialmente carcinogênicos ${ }^{5,2}$.

A GCA é uma conseqüência tardia da infecção pelo Helicobacter pylori (Hp) em aproximadamente 1/ 3 dos pacientes infectados. Com base nesta afirmativa tem-se sugerido que o CG desenvolve-se menos freqüentemente naqueles pacientes tratados. Estudos recentes têm demonstrado que o uso prolongado dos IBP estaria associado a uma piora progressiva da gastrite do corpo em pacientes infectados pelo Hp. Esta observação tem indicado a erradicação do Hp antes de se iniciar o tratamento a longo prazo pelo IBP para a DRGE e outras doenças relacionados a hipercloridria gástrica ${ }^{6}$.

Além do comprometimento das células parietais, a atrofia da mucosa gástrica, sobretudo a do fundo e do corpo, reduz o número de células principais (zimogênicas) com conseqüente comprometimento da produção de pepsinogênios As funções destas células são comprometidas pela supressão de genes codificadores da $\mathrm{H}^{+} \mathrm{K}^{+} / \mathrm{ATP}$ ase e do pepsinogênio, nos regimes de hipocloridria acentuada. PERASO \& TESTINO (1991) relataram que os baixos níveis séricos de pepsinogênio dos tipos 1 e 2 seriam preditores de alterações histológicas da mucosa gástrica, como as PAPG ("pepsinogen altered pyloric glands") que estariam muito relacionados com a gravidade da $\mathrm{GCA}^{7}$. Estudo recente concluiu que as células parietais produzem sinais reguladores que controlariam o programa de diferenciação celular de linhagens de células principais, sugerindo que a redução na população de células parietais, na gastrite atrófica, poderia comprometer a diferenciação das células principais ${ }^{8}$.

Apesar do grande número de publicações recentes sobre o valor da dosagem do pepsinogênio sérico e da identificação de PAPG, como métodos de rastreamento da GCA e do CG, não se encontrou na literatura nenhum trabalho em que se relacionasse a população e distribuição das células principais com o uso prolongado de inibidores de bomba de prótons ${ }^{7}$. Encontraram-se, alguns estudos histopatológicos relacionando a população de células parietais ou a sua atividade secretora, com a extensão e desenvolvimento da GCA e do CG ${ }^{9,10,11}$. Poucos estudos, entretanto, relacionaram estes parâmetros com o uso prolongado dos IBP ${ }^{4}$.

Assim sendo, o objetivo deste trabalho foi estudar o efeito do uso de pantoprazol, por 15 semanas, associado ou não a carcinogênese pela NMU, sobre o estômago glandular de ratos Wistar, através da análise histológica e computadorizada das áreas com células parietais (AP), principais (AZ) e da mucosa não oxíntica (ANO), além do estudo das alterações histopatológicas identificadas.

\section{MÉTODOS}

Foram utilizados 40 ratos Wistar, machos, recémdesmamados, com peso médio de $80 \mathrm{~g}$, todos procedentes do Centro de Bioterismo do Instituto de Ciências Biológicas da UFMG. Os animais foram acondicionados em gaiolas de poliuretano, forradas com camada de $5 \mathrm{~cm}$ a $10 \mathrm{~cm}$ de serragem e mantidas à temperatura 
ambiente, com fotoperíodo de 12 horas (claro/escuro). Os mesmos foram observados diariamente, por 15 semanas, para se verificar a oferta de água e ração (NOVILAB/Mercavet S/A), além da administração das drogas. Com base no tratamento, os animais foram divididos em quatro grupos de 10 ratos cada: G1 (Controle), G2 (PTZ + NMU), G3 (PTZ) e G4 (NMU).

O pantoprazol (Bik Química e Farmacêutica Ltda) foi adquirido em ampolas de 40mg/10ml, e administrado a G2 e G3 através de injeções intra-peritoneais na dose de $14 \mathrm{mg} / \mathrm{kg}$ de peso (2x/semana). Para a carcinogênese foi utilizada a NMU $\left(\mathrm{C}_{2} \mathrm{H}_{5} \mathrm{~N}_{3} \mathrm{O}_{2}\right)$, frasco de $25 \mathrm{~g}$ ( ${ }^{\circ}$ N4766/ Sigma-Aldrich do Brasil), oferecida a G2 e

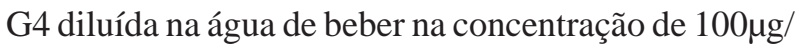
$\mathrm{ml}(100 \mathrm{ppm} / \mathrm{ml})$, ad libitum ${ }^{12}$.

Os ratos foram operados na $15^{\text {a }}$ semana do experimento. A técnica anestésica consistiu na utilização da associação de $90 \mathrm{mg} / \mathrm{kg}$ de peso de cloridrato de cetamida (Lab. Parke-Davis) com $15 \mathrm{mg} / \mathrm{kg}$ de peso de xilazina (Lab. Virbac). Após laparotomia mediana ampla, procedeu-se à retirada do estômago, seccionando-se os ligamentos gástricos, vasos da curvatura menor, esôfago distal e bulbo duodenal proximal. A eutanásia foi realizada ainda com o animal anestesiado, por secção da aorta abdominal. A seguir, os estômagos foram abertos e, cuidadosamente, fixados sem tensão sobre uma placa de isopor. O conjunto foi colocado, posteriormente, dentro de um Becker de $500 \mathrm{ml}$ contendo solução de formaldeído a $10 \%$, por cerca de $24 \mathrm{~h}$. Posteriormente os estômagos foram separados da placa de isopor, colocados sobre uma superfície de borracha dura, onde foram cortados em 10 fragmentos medindo $5 \mathrm{~mm}$ de largura cada. A seguir, os estômagos foram fotografados ("Canon" EOS 620) e, posteriormente, ressecado o segmento membranoso, permanecendo os fragmentos individualizados com pequena área de mucosa membranosa (epitélio escamoso) com cerca de $1 \mathrm{~mm}$ de largura, para orientar o estudo histológico. Os fragmentos foram numerados da esquerda para a direita com o segmento membranoso em posição cranial 3. O comprimento total do fragmento (FT) foi medido, em milímetros, com o auxílio de marcas milimetradas presentes na platina (mesa) do microscópio ótico utilizado (Nikon/Alpha-phot/2452-H). Da mesma maneira foram obtidos, de cada fragmento estudado, os comprimentos correspondentes à extensão de mucosa com células parietais (CP) e com células principais (CZ). Conhecendo-se as medidas de FT, CP e CZ, do mesmo fragmento, calcularam-se os valores percentuais de $\mathrm{CP}$ e $\mathrm{CZ}$, em ralação à medida total do fragmento (FT) que seria igual a $100 \%$.
No estudo histopatológico considerou-se a presença de atrofia, metaplasia, displasia e inflamação crônica, inflamação aguda (atividade) e carcinoma. As alterações inflamatórias foram classificadas em aguda (atividade) ou crônica, segundo os critérios da classificação de Sydney modificada ${ }^{13}$. Considerou-se a presença de atrofia glandular quando havia perda de tecido glandular e adelgaçamento da mucosa. O critério para a identificação de metaplasia seria a presença de células intestinais ou pancreáticas. Quanto às presenças de displasia e câncer no epitélio glandular, procurou-se atipia nuclear. As alterações encontradas foram classificadas em leve, moderadas ou acentuadas. Quanto à região acometida, considerou-se o acometimento restrito à mucosa do corpo e/ou do antro.

As fotos dos estômagos formalizados e cortados foram, inicialmente, digitalizadas e copiadas (CanoScan/ FB320P) numa resolução gráfica de 300DPI e tamanho $100 \%$ do original. Utilizando o programa de computação gráfica "ImageJ 1.19z", foi feita a marcação de FT de todos os 10 fragmentos de cada um dos 40 estômagos estudados. Com recursos de magnificação de imagem do programa em questão foi possível identificar diferenças de relevo e coloração entre os tipos de mucosa estudados ${ }^{11,14}$. O programa atribuía uma medida à marcação de FT, em unidades digitais denominadas "pixel" (1 "pixel" = 0,09 mm).

Considerando os valores proporcionais obtidos a partir do estudo histológico, bem como a previa marcação e medida de FT, procedeu-se o cálculo dos valores de CP e CZ, em "pixels" para cada fragmento. Em seguida, CP e CZ foram demarcados através de traços longitudinais de cor vermelha e azul, respectivamente. O ponto de união dessas linhas com a borda direita de cada fragmento, foi identificado e utilizado como um ponto de demarcação das áreas a serem estudadas. Os fragmentos de numero 10 foram demarcados de forma diferente, uma vez que suas bordas direitas se apresentavam de forma irregular. Assim sendo, para estes fragmentos o corte histológico utilizado foi obtido da metade de sua espessura. O contorno do segmento glandular determinava sua área total (AT). A união de todos os pontos de demarcação de CP com o contorno proximal da mucosa glandular determinava a área com células parietais (AP). De forma semelhante, a união de todos os pontos de demarção de CZ com o contorno proximal da mucosa glandular, determinava a área com células principais (AZ) (Figura 1). A unidade gráfica digital utilizada para as medidas de área, foi o "square pixel". A partir desta medida calculou-se os valores percentuais de AP, AZ e ANO, em relação à AT (Figura 2). 


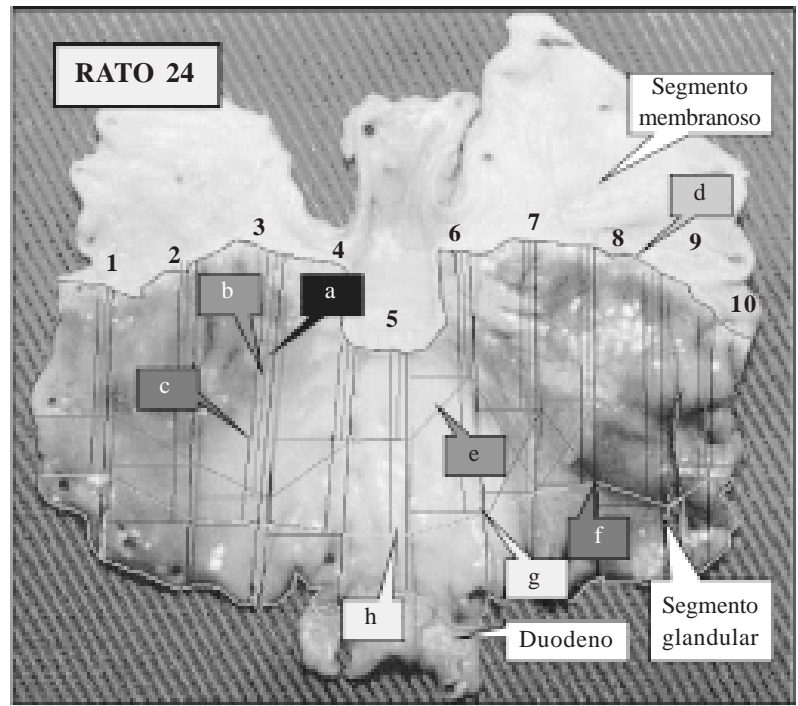

Figura 1 - Exemplo de medidas gerais do estudo computadorizado dos segmentos gástricos glandulares. a) linha preta (FT), b) linha vermelha (CP), c) linha azul (CZ), d) linha de contorno da área de AT, e) ponto de demarcação da área de AP, f) ponto de demarcação da $\mathrm{AZ}, \mathrm{g}$ ) ponto de demarcação da $\mathrm{ANO}, \mathrm{h}$ ) linha de contorno da ANO, números de 1 a 10: fragmentos estudados.

As comparações entre os quatro grupos estudados em relação às variáveis computadorizadas foram realizadas utilizando-se o teste de Kruskal-Wallis. Todos os resultados foram considerados significativos para uma probabilidade de significância inferior a $5 \%(\mathrm{p}<0,05)$.

\section{RESULTADOS}

$\mathrm{Na}$ análise dos resultados foram considerados as variáveis computadorizadas (AZ, AP e ANO) e os achados histopatológicos. As medidas descritivas das variáveis computadorizadas foram apresentadas em valores percentuais relativos aos valores em "pixels" das medidas de AT (100\%). Na tabela 1 foram apresentados valores de mediana, média, mínimo (mín), máximo (máx) e desvio padrão (dp). O valor de n entre parên-

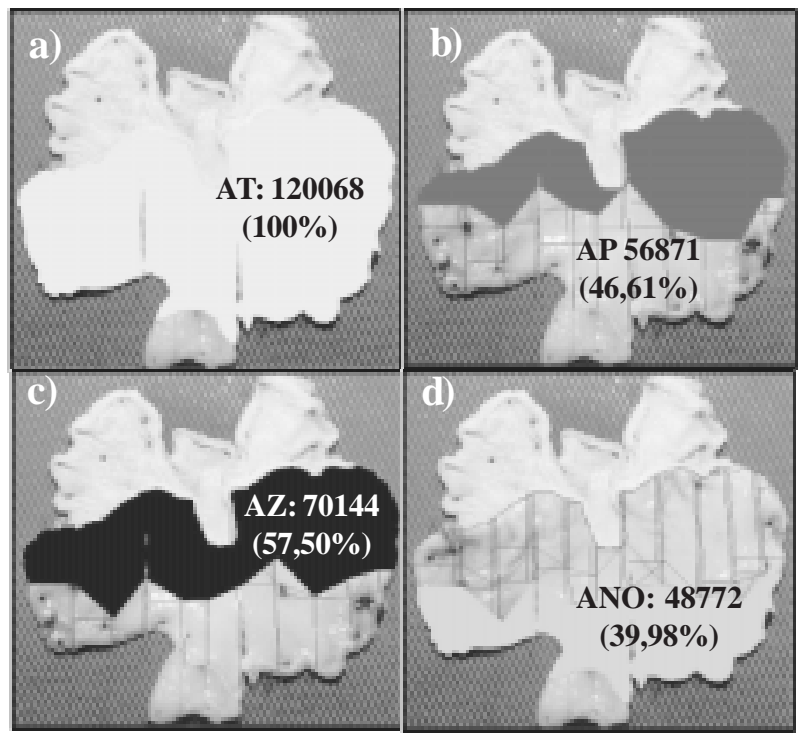

Figura 2 - Exemplos de medidas de área do segmento gástrico glandular (Rato 25 - G2: NMU + PTZ): a) área total (AT); b) área com células parietais (AP); c) área com células principais (AZ) e d) área de mucosa não oxíntica (ANO).

teses nos gráficos referiu-se ao tamanho da amostra avaliada.

Realizou-se um estudo comparativo entre os quatro grupos estudados em relação às medidas das variáveis computadorizadas. Observou-se uma diferença significativa $(p<0,001)$ entre os grupos em relação às medidas das variáveis computadorizadas AP e ANO. Para a variável AP o grupo controle apresentou resultados significativamente superiores aos outros três grupos e esses foram similares entre si. Situação inversa foi observada com relação à variável ANO, onde, o grupo controle apresentou medidas significativamente inferiores aos demais que, por sua vez, foram semelhantes entre si. Quanto a variável AZ, as medidas foram semelhantes nos quatro grupos, sem significância estatística (Tabela 1).

Tabela 1 - Comparação dos grupos estudados em relação as variáveis computadorizadas.

\begin{tabular}{|c|c|c|c|c|c|c|c|}
\hline \multirow[b]{2}{*}{ Variável } & \multirow[b]{2}{*}{ Grupo } & \multicolumn{5}{|c|}{ Medidas descritivas } & \multirow[b]{2}{*}{ p } \\
\hline & & Mínimo & Máximo & Mediana & Média & dp & \\
\hline \multirow{4}{*}{$\mathbf{A P}$} & Controle & 66,6 & 85,9 & 74,9 & 76,8 & 5,9 & $<0,001$ \\
\hline & $\mathrm{NMU}+\mathrm{PTZ}$ & 45,0 & 68,8 & 57,3 & 56,2 & 7,9 & \\
\hline & PTZ & 46,6 & 58,0 & 54,8 & 53,3 & 3,7 & $1>(2=3=4)$ \\
\hline & NMU & 49,2 & 59,3 & 52,0 & 52,9 & 3,3 & \\
\hline \multirow{4}{*}{$\mathbf{A Z}$} & Controle & 43,5 & 83,0 & 55,2 & 59,9 & 14,9 & 0,493 \\
\hline & $\mathrm{NMU}+\mathrm{PTZ}$ & 48,7 & 67,3 & 57,1 & 58,1 & 6,4 & \\
\hline & PTZ & 53,7 & 74,8 & 58,9 & 61,4 & 6,3 & $(1=2=3=4)$ \\
\hline & NMU & 53,7 & 67,9 & 63,6 & 62,2 & 4,5 & \\
\hline \multirow{4}{*}{ ANO } & Controle & 11,8 & 31,0 & 23,1 & 21,2 & 6,6 & $<0,001$ \\
\hline & $\mathrm{NMU}+\mathrm{PTZ}$ & 28,5 & 46,8 & 35,5 & 36,4 & 7,1 & \\
\hline & PTZ & 26,5 & 40,6 & 36,0 & 35,3 & 4,4 & $(2=3=4)>1$ \\
\hline & NMU & 30,3 & 43,2 & 33,6 & 35,3 & 4,1 & \\
\hline
\end{tabular}

Nota: $\mathrm{O}$ valor de $\mathbf{p}$ na tabela refere-se ao teste de Kruskal-Wallis

$\begin{array}{lrl}\text { Legenda: } 1 \rightarrow \text { Controle } & 2 \rightarrow \text { NMU + PTZ } \quad 3 \rightarrow \text { PTZ } & 4 \rightarrow \text { NMU }\end{array}$ 
Das alterações histopatológicas pesquisadas, ou seja: atrofia, displasia, carcinoma, inflamação crônica e inflamação aguda (atividade), somente foram encontrados casos de atrofia, inflamação crônica e inflamação aguda. As tabelas 2, 3 e 4 apresentam a distribuição por grupo dos achados histopatológicos encontradas. Quanto à presença ou não de determinado achado o número de casos impediu a comparação entre os grupos estudados. Não se realizou, portanto, nenhuma comparação entre os achados histopatológicos e as variáveis computadorizadas.

Tabela 2 - Caracterização dos grupos estudados quanto à presença de atrofia.

\begin{tabular}{l|c|c|c|c|c|c}
\hline & \multicolumn{9}{|c|}{ Atrofia } & \multicolumn{3}{c}{} \\
\cline { 2 - 7 } Grupo & \multicolumn{2}{|c|}{ Ausente } & \multicolumn{2}{c|}{ Presente } & \multicolumn{2}{c}{ Total } \\
\cline { 2 - 7 } & $\mathbf{n}$ & $\boldsymbol{\%}$ & $\mathbf{N}$ & $\mathbf{\%}$ & $\mathbf{1 0}$ \\
\hline Controle & 9 & 90,0 & 1 & 10,0 & $\mathbf{1 0}$ & $\mathbf{1 0 0 , 0}$ \\
NMU + PTZ & 7 & 70,0 & 3 & 30,0 & $\mathbf{1 0}$ & $\mathbf{1 0 0 , 0}$ \\
PTZ & 6 & 60,0 & 4 & 40,0 & $\mathbf{1 0}$ & $\mathbf{1 0 0 , 0}$ \\
NMU & 10 & 100,0 & 0 & 0,0 & $\mathbf{1 0}$ & $\mathbf{1 0 0 , 0}$ \\
\hline
\end{tabular}

Tabela 3 - Caracterização dos grupos estudados quanto à presença de inflamação crônica.

\begin{tabular}{|c|c|c|c|c|c|c|}
\hline \multirow[b]{3}{*}{ Grupo } & \multicolumn{4}{|c|}{ Inflamação crônica } & & \\
\hline & \multicolumn{2}{|c|}{ Ausente } & \multicolumn{2}{|c|}{ Presente } & \multicolumn{2}{|c|}{ Total } \\
\hline & $\mathbf{n}$ & $\%$ & $\mathbf{N}$ & $\%$ & $\mathbf{n}$ & $\%$ \\
\hline Controle & 4 & 40,0 & 6 & 60,0 & 10 & 100,0 \\
\hline $\mathrm{NMU}+\mathrm{PTZ}$ & 4 & 40,0 & 6 & 60,0 & 10 & 100,0 \\
\hline PTZ & 10 & 100,0 & 0 & 0,0 & 10 & 100,0 \\
\hline NMU & 5 & 50,0 & 5 & 50,0 & 10 & 100,0 \\
\hline
\end{tabular}

Tabela 4 - Caracterização dos grupos estudados quanto à presença de inflamação aguda (atividade).

\begin{tabular}{l|c|c|c|c|c|c}
\hline & \multicolumn{9}{|c|}{ Atividade } & \multicolumn{3}{c}{ Total } \\
\cline { 2 - 7 } Grupo & \multicolumn{2}{|c|}{ Ausente } & \multicolumn{2}{c|}{ Presente } & \multicolumn{2}{c}{} \\
\cline { 2 - 7 } & N & \% & n & \% & n \\
\hline Controle & 4 & 40,0 & 6 & 60,0 & $\mathbf{1 0}$ & $\mathbf{1 0 0 , 0}$ \\
NMU + PTZ & 4 & 40,0 & 6 & 60,0 & $\mathbf{1 0}$ & $\mathbf{1 0 0 , 0}$ \\
PTZ & 10 & 100,0 & 0 & 0,0 & $\mathbf{1 0}$ & $\mathbf{1 0 0 , 0}$ \\
NMU & 7 & 70,0 & 3 & 30,0 & $\mathbf{1 0}$ & $\mathbf{1 0 0 , 0}$ \\
\hline
\end{tabular}

\section{DISCUSSÄO}

Tão logo os IBP tornaram-se disponíveis iniciouse uma discussão sobre os possíveis riscos decorrentes de seu uso prolongado. Segundo alguns autores, ao contrário do que ocorre em animais, a hipergastrinemia não seria um problema relevante no homem, e um aumento dos níveis de gastrina durante o tratamento com os IBP pode induzir a uma proliferação das células gástricas endócrinas em alguns casos. Estudos controlados com casuísticas maiores de pacientes em longo período de tratamento com IBP seriam, entretanto necessários para recomendar, sem receio, o uso desta classe de medicamentos.

Optamos por utilizar o pantoprazol e não outro IBP, por não termos encontrado na literatura nenhum estudo relacionando seu uso crônico com o desenvolvimento de GCA ou câncer gástrico. Inicialmente pretendia-se utiliza-lo por via oral, na dose $4 \mathrm{mg} / \mathrm{Kg} / \mathrm{dia}$ ${ }^{3}$. Verificou-se, entretanto, que ao se diluir o medicamento na água de beber após maceração dos comprimidos, este precipitava ou aderia às paredes do recipiente. Pensou-se, então, em administrar o preparado 
por intubação nasogástrica de cada animal. Tal procedimento mostrou-se pouco prático além de não garantir que animal ingerisse a dose preconizada do medicamento, uma vez que parte deste permanecia aderido ao cateter. Optou-se, então, por injeções intra-peritoneais de $14 \mathrm{mg} / \mathrm{kg}$ (2x por semana), correspondente à dose de $4 \mathrm{mg} / \mathrm{Kg} /$ dia preconizada por ROHR et al. (1992) ${ }^{3}$, garantindo-se assim que todos os animais fizessem uso da dose preconizada do medicamento, considerandose seu peso exato que era aferido no momento da aplicação.

A opção pela linhagem Wistar se deveu à experiência prévia de outros autores que realizaram estudos semelhantes ao nosso, bem como a dificuldade em se adquirir outra linhagem em nosso meio. Quanto à opção por machos, considerou-se a maior resistência destes a ação dos compostos N-nitrosos, evitando-se que os mesmos viessem a morrer antes do término do experimento ${ }^{3,12}$. Durante todo o experimento, os 4 grupos de 10 animais cada foram mantidos na mesma gaiola.

Para que se pudesse avaliar o efeito do pantoprazol sobre a distribuição das células parietais e zimogênicas, bem como relacionar este efeito com alterações histopatológicas que porventura surgissem, foi necessário utilizar um agente com reconhecida ação carcinogênica sobre a mucosa gástrica, objetivando induzir o aparecimento dessas alterações. Assim sendo, recorremos a literatura e identificamos estudos recentes que utilizaram a NMU para a carcinogênese gástrica experimental, com indução de lesões pré-neoplásicas e tumores no segmento glandular do estômago de ratos. Não era objetivo deste estudo promover o aparecimento de tumores ou relacioná-los qualitativa e quantitativamente com o uso do pantoprazol, mas assim criar um ambiente propício ao desenvolvimento de lesões pré-neoplásicas, para que pudéssemos estudar o efeito deste medicamento sobre a mucosa gástrica glandular do rato. Optou-se então, pela menor dosagem e menor tempo de ingestão da NMU, que segundo a literatura promoveriam alterações histopatológicas na mucosa gástrica glandular do rato ${ }^{12}$.

Com o objetivo de se avaliar a distribuição das células parietais e principais na mucosa glandular do estômago de ratos, optou-se por um estudo computadorizado das áreas ocupadas por estas células em relação à área total do segmento glandular. Este estudo foi possibilitado pela análise dos dados fornecidos pelo estudo histológicos, referentes às medidas dos fragmentos de cada um dos 40 estômagos estudados. Para tanto, utilizou-se a versão de domínio público do programa "NIH Image" ("National Institutes of Health, Bethesda, MD, USA"), denominado "ImageJ 1.19z", que pode ser obtido gratuitamente no endereço eletrônico: http://rsb.info.nih.gov/ij/. Através de recursos de magnificação de imagem, análise de textura e tonalidades de cores, este programa possibilita a realização de estudos morfométricos sistematizados além de medidas diversas de imagens digitalizadas. $\mathrm{O}$ "NIH Image" e suas versões têm sido muito utilizados nos mais variados estudos, para se avaliar número, área, volume, diâmetro e forma de células ou conjunto celulares, ou para se analisar qualitativa e quantitativamente a presença de compostos intra ou extracelulares com o auxílio de técnicas de imunohistoquímica, a partir do estudo de imagens digitalizadas ${ }^{11,14}$.

Comparando-se os grupos estudados em relação as variáveis computadorizadas, observou-se uma diferença significativa entre os grupos em relação às medidas das variáveis AP e ANO $(\mathrm{p}<0,05)$. Segundo estes dados, o uso contínuo de pantoprazol como de NMU por 15 semanas, isoladamente ou em associação, levou a uma redução de AP e aumento de ANO no segmento gástrico glandular de ratos Wistar machos.

Quanto à relação entre os grupos estudados e os achados histopatológicos, somente foram encontrados casos com atrofia, inflamação crônica e atividade, todos em número que não possibilitou o emprego de nenhum tratamento estatístico. Supomos que um estudo por tempo mais prolongado, sobretudo com a administração de maiores doses de NMU, possa levar ao aparecimento de alterações histopatológicos em numero tal que possa permitir uma comparação estatística em os grupos estudados ${ }^{12}$.

O prosseguimento desta linha de pesquisa nos permitirá conhecer melhor o efeito do uso prolongado dos IBP sobre a mucosa gástrica.

Concluímos que tanto o pantoprazol aplicado por via peritoneal na dose semanal de $40 \mathrm{mg} / \mathrm{Kg}$ peso, duas vezes por semana, quanto a NMU diluída $(100 \mu \mathrm{g} / \mathrm{ml})$ na água de beber, oferecida ad libitum, isoladamente ou ministrados associadamente, quando empregados por 15 semanas, promoveram em proporções semelhantes, redução da área com células parietais e aumento da mucosa oxíntica em ratos Wistar machos.

\section{REFERÊNCIAS}

1. Módena JLP, Pereira LCC. Carcinoma gástrico precoce: In: SOBED, Endoscopia digestiva. 3ed. Rio de Janeiro: MEDSI; 2000. p 402-27.

2. Mowat AT C, Carswell A, Wirz A, McColl KE. Omeprazole and dietary nitrate independently affect levels of vitamin $\mathrm{C}$ and nitrite in gastric juice. Gastroenterology 1999;116:813-22.

3. Rohr I, Ockert D, Reznik GK. Ultrastructural investigations of the enterochromafin-like (ECL) cells in three different rat strains after treatment with the $\mathrm{H}^{+} \mathrm{K}^{+}$/ATPase inhibitor pantoprazole. Exp Toxic Pathol 1992; 44:197-200.

4. Mccloy RF, Arnold R, Bardhan KD, Cattan D, KlinkenbergKnol E, Maton PN, Riddell RH, Sipponen P. Pathophysiological 
effects of long-term acid suppression in man. Dig Dis Sci 1995;40:116-20.

5. Calmels S, Bereziat JC, Ohsihma H, Bartsch H. Bacterial formation of $\mathrm{N}$-nitroso compounds in the rat stomach after omeprazole-induced achlorhydria. IARC Sci Publ 1991;105:187-91.

6. Kokkola A, Rautelin H, Puolakkainen P, Sipponen P, Farkkila M, Haapianen R, Kosunen TU. Diagnosis of Helicobacter pylori infection in patients with atrophic gastritis. Scand J Gastroenterol 2000;35:138-41.

7. Peraso A, Testino G. Advanced gastric cancer of the antrum: anatomic-functional correlation between chief cell mass and serum pepsinogen. Gastroenterol Jpn 1991;26:588-92.

8. Karam SM. Cell lineage relationship in the stomach of normal and genetically manipulated mice. Braz J Med Biol Res 1998;31:271-9.

9. Fujishima K, Misumi A, Akagi M. Histophatologic study on development and extension of atrophic change in the gastric mucosa. Gastroenterol Japonica 1984;19:9-17.
10. Misumi A, Yagi Y, Harada K, Honmyo U, Murakami A, Takano S, Mizumoto S. Relationship between atrophic gastritis classified by parietal cell condition and gastric acid secretion. Kumamoto Med J 1987;40:129-38.

11. Miyazaki SM, Matsuda MG, Misumi A, Honmyo U, Murata H, Sagara K, Kurano R, Okabe H. Blood flow, acidity and atrophic change of gastric mucosa in Mongolian gerbils infected by Helicobacter pylori. Digestive Endoscopy 2001 (in print).

12 Watanabe H, Ando Y, Yamada K, Okamoto T, Ito A. Lack of any positive effect of intestinal metaplasia on induction of gastric tumors in Wistar rats treated with N-methyl-Nnitrosourea in their drinking water. Jpn J Cancer Res 1994;85:892-6.

13. Dixon MF, Path FRC, Genta RM. Classification and grading of gastritis, the updated Sydney system. Am J Surg Pathol 1996;20:1161-81.

14. White SL, Smith WC, Fisher LF, Gatlin CL, Hanansono GK, Jordan WH. Quantification of glandular gastric changes in rats given a proton pump inhibitor for 3 months with emphasis on sampling scheme selection. Toxicol Pathol 1998;26:403-10.

Souza IKF, Lázaro da Silva A, Misumi A, Savi D, Custódio DS, Maia FFR, Canela GGC, Oliveira CHC. Histological and computer-assisted analysis of parietal and chief cells stomach areas in Wistar rats treated with pantoprazole and N-Nitroso-N-Methylurea (NMU). Acta Cir Bras [serial online] 2002 Jul-Aug;17(4). Available from URL: http://www.scielo.br/acb.

ABSTRACT - The long-term use of proton bomb inhibitors has been considered a risk condition for the development of atrophic gastritis and gastric tumors. Objective: The aim of this study was to investigate the effects of pantoprazole (PTZ) treatment and N-Nitroso-N-Methylurea (NMU) carcinogenesis, for 15 weeks, in the glandular stomach of rats by histological and computerassisted analysis of parietal cells area (PA), chief cells area (CP) and non-oxintic mucosal area (ANO), as well as by histopathological study. Methods: A total of 40 male Wistar rats were divided into four groups on the basis of the treatment: G1 (control), G2 (NMU+PTZ), G3 (PTZ) and G4 (NMU). Pantoprazole was administered twice a week $(14 \mathrm{mg} / \mathrm{kg}$ body wt., i.p.) and NMU was given in the drinking water (100ppm) ad libitum. After histological examination AP, AZ and ANO were investigated by computer-assisted analysis of the stomach image using the program ImageJ1.19z. Results: Showed a reduction of AP and increase of ANO in G2, G3 and G4 (p<0,001). Cases of atrophy, acute and chronic inflammation were found in number that impeded statistical comparison among the studied groups. Conclusion: The continuous administration, for 15 weeks, of pantoprazole (14mg/kg body wt., i.p.) and NMU (100ppm in the drinking water, ad libitum), isolated or associated, determines a reduction of parietal cells area and increase of non-oxintic mucosal area in glandular stomach of male Wistar rats.

KEY WORDS: Pantoprazole. Methylnitrosourea. Stomach neoplasms. Gastritis. Atrophy.

\section{Endereço para correspondência:}

Conflito de interesse: nenhum

Fonte de financiamento: $\mathrm{CNPq}$

Alcino Lázaro da Silva

Rua Guaratinga, 151.

30315-430 Belo Horizonte - MG

Fone: (31)221-3072

Data do recebimento: $12 / 03 / 2002$

Data da revisão: 22/04/2002

Data da aprovação: 18/05/2002 